Original Article

\title{
Use of a visual feedback-equipped reacher in reach-to-grasp movements
}

\author{
Ji-Hye Do ${ }^{1)}$, Won-GYU Yoo ${ }^{2 *}$ \\ 1) Department of Occupational Therapy, Kijeon College, Republic of Korea \\ 2) Department of Physical Therapy, College of Healthcare Medical Science and Engineering, \\ Inje University: 607 Obangdong, Gimhae, Gyeongsangnam-do 621-7849, Republic of Korea.
}

\begin{abstract}
Purpose] The purpose of this study was to investigate the effectiveness of using a visual feedbackequipped reacher when performing reach-to-grasp tasks. [Subjects and Methods] Seventeen healthy participants with no illnesses or physical disabilities were selected for this study. Activation of the flexor digitorum superficialis and extensor digitorum muscles was assessed using electromyography. Wrist and shoulder momentums were calculated using a three-axis acceleration sensor. Participants were instructed to move 10 ears of plastic corn from right to left using a reacher with and without visual feedback. [Results] Comparison of muscle activity under the two conditions revealed a statistically significant reduction in the activity of the FDS and ED with feedback compared to without. The three-axis accelerometer values showed that wrist momentum decreased significantly with visual feedback compared to without feedback. [Conclusion] The results showed that using the vision-assisted reacher affected upper limb muscle activation and momentum. It is important that assistive devices be developed for effective energy use. Here, providing visual feedback during reacher use was shown to reduce users' physical fatigue. Key words: Accelerometer, Electromyography, Reacher
\end{abstract}

(This article was submitted Nov. 6, 2017, and was accepted Nov. 18, 2017)

\section{INTRODUCTION}

A reacher had frequently been used to supplement limited upper extremity and trunk motions ${ }^{1)}$. Visual feedback is important for adequate motor control, but the reacher provides insufficient visual feedback. Furthermore, this limited information may be further compounded by task complexity and the shape of the object ${ }^{1)}$. Visual information is very important for object manipulation when using an assistive device because skillful use of these devices depends on information about the object's shape and orientation. Some studies have demonstrated the importance of perception in locating objects, and others have found that information about object location is key in determining the initial hand position, and is related to anticipatory reaching and grasping movements ${ }^{2}$. Appropriate assistive devices must be chosen to help patients engage in their occupations and participate in their communities ${ }^{3}$. Occupational therapists must understand the interaction between abilities and the environment, and perform professional evaluations and make recommendations regarding assistive devices for clients with disabilities ${ }^{3)}$. Considerable research is still needed to ensure the appropriate use of assistive devices. However, to date research has not produced clear results. The purpose of the present study was to use electromyography (EMG) and three-axis accelerometer data to investigate the effects of visual feedback on the muscle activity and momentum of the upper limb during use of a visual feedback-equipped reacher.

\section{SUBJECTS AND METHODS}

Seventeen college students aged 20-24 years participated in this study. No participant reported musculoskeletal problems or current use of a reacher. The purpose and methods of the study were explained to the subjects, and written informed

*Corresponding author. Won-gyu Yoo (E-mail: won7y@inje.ac.kr)

(C2018 The Society of Physical Therapy Science. Published by IPEC Inc.

(c) $(-)$ This is an open-access article distributed under the terms of the Creative Commons Attribution Non-Commercial No Deriva-

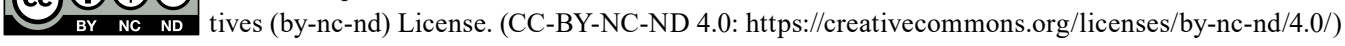


consent was obtained, in keeping with the ethical principles of the Declaration of Helsinki. Participants were instructed to move 10 ears of plastic corn from right to left using a reacher with and without visual feedback. The assessment conditions were designed to identify differences in muscle activation between using and not using the reacher during the performance of our 'static corn task'. This task requires that the participant moves an ear of corn from right to left using a reacher; the task is repeated 10 times. The reacher was designed to either provide or not provide feedback. The direction and distance of movement were calculated based on the initial hand and object positions. The static corn was aligned with the midpoint of the participant's trunk, at a distance (based on arm length) such that the participant could reach and grasp the corn without moving the trunk. For noninvasive surface EMG, we used an automated EMG system (Biopack Systems, Inc., USA). To quantify muscle activity during reaching, bipolar EMG electrodes were placed over the flexor digitorum superficialis (FDS) and extensor indicis muscles. The gain was set at 1,000, with a common mode rejection ratio of $90 \mathrm{~dB}$. The triaxial accelerometer (EMG Systems, Brazil) used in this study $(0.7 \mathrm{~cm}$ high $\times 2 \mathrm{~cm}$ long $\times 1.6 \mathrm{~cm}$ wide; weight, approximately 25 grams) was securely taped to the dorsal face of the forearm, between the styloid process of the ulna and the radius. Momentum was analyzed in terms of signal vector magnitude (SVM) based on data obtained from an acceleration sensor. Statistical analysis was performed using SPSS software (ver. 24.0; SPSS Inc., USA), and statistical significance was defined as $p<0.05$. The experimental conditions were compared using the Wilcoxon signed-rank test, a nonparametric statistical method.

\section{RESULTS}

Comparison of muscle activity revealed a statistically significant difference between conditions, with activity decreasing under the visual feedback condition, from $89.5 \pm 35.2 \%$ to $59.4 \pm 22.1 \%$ and from $65.9 \pm 28.9 \%$ to $48.0 \pm 19.4 \%$ ( $<<0.05$ ) in the FDS and extensor indicis muscles, respectively. In the case of the momentum, the three-axis SVM values showed that wrist momentum decreased significantly with visual feedback, from $5,420 \pm 2,286 \mathrm{~cm} / \mathrm{s}^{2}$ to $4,356 \pm 1,973 \mathrm{~cm} / \mathrm{s}^{2}(\mathrm{p}<0.05)$, compared to values without visual feedback. Shoulder momentum did not differ significantly between conditions $(4,226 \pm$ $1,848 \mathrm{~cm} / \mathrm{s}^{2}$ without vs. $3,602 \pm 1,546 \mathrm{~cm} / \mathrm{s}^{2}$ with visual feedback).

\section{DISCUSSION}

This study results confirm that muscle activation and movement momentum differ when patients use a visual feedbackequipped reacher instead of a non-equipped reacher. This result supports previous studies showing that visual feedback affects movement accuracy during targeting tasks ${ }^{3)}$. When such feedback was provided, muscle activity tended to decrease compared to when no feedback was available. In the case of momentum, the three-axis SVM values showed that wrist momentum decreased significantly with visual feedback compared to without. If the use of an inappropriate assistive device leads to abnormal wrist movement, other musculoskeletal problems may result. The accelerometer data also showed that participants' momentum was more appropriate to the task when they used the reacher with visual feedback. Thus, these results indicated that using the feedback-equipped reacher led to a novel pattern of movement that required low energy expenditure $^{4)}$. Visual information is very important for object manipulation when using an assistive device because skillful use of these devices depends on information about the object's shape and orientation ${ }^{5}$. The results represent an important first step in the development of reachers equipped with visual feedback. It is important to note that during use, a reacher should not continually produce abnormal movements. One important goal of this initial study was to determine the reacher types that are most appropriate for patient use. This study had some limitations that should be noted. First, this study was conducted in a laboratory setting with a healthy population. Thus, the results may not be generalizable to normal day-to-day activities. Further studies should include motion analysis and other techniques for studying wrist musculature and movement.

\section{Conflict of interest}

None.

\section{REFERENCES}

1) Pinkston NE, Boersma AJ, Spaulding SJ: The impact of reacher length on electromyography activity and task. Can J Occup Ther, 2005, 72: 89-95. [Medline] [CrossRef]

2) Itaguchi Y, Fukuzawa K: Hand-use and tool-use in grasping control. Exp Brain Res, 2014, 232: 3613-3622. [Medline] [CrossRef]

3) Maitra KK, Philips K, Rice MS: Grasping naturally versus grasping with a reacher in people without disability: motor control and muscle activation differences. Am J Occup Ther, 2010, 64: 95-104. [Medline] [CrossRef]

4) Yoo IG, Jung MY, Jeon HS, et al.: Effects of wrist-extension orthosis on shoulder and scapular muscle activities during simulated assembly tasks. Ind Health, 2010, 48: 108-114. [Medline] [CrossRef]

5) Maruishi M, Tanaka Y, Muranaka H, et al.: Brain activation during manipulation of the myoelectric prosthetic hand: a functional magnetic resonance imaging study. Neuroimage, 2004, 21: 1604-1611. [Medline] [CrossRef] 